\title{
MELODA, MÉTRICA PARA EVALUAR LA REUTILIZACIÓN DE DATOS ABIERTOS
}

\section{Alberto Abella, Marta Ortiz-de-Urbina-Criado y Carmen De-Pablos-Heredero}

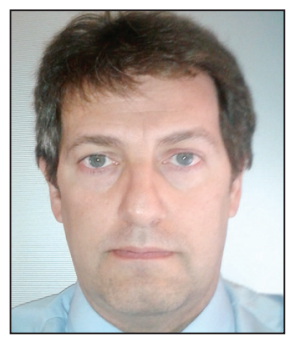

Alberto Abella es ingeniero superior de telecomunicaciones, profesor asociado en la Universidad Rey Juan Carlos y estudiante de la Escuela Internacional de Doctorado en el Programa de Ciencias Sociales y Jurídicas en la Universidad Rey Juan Carlos. Es experto en los campos de open data y open government aplicados al sector público y privado. Es presidente de la Open Knowledge Foundation en España.

http://orcid.org/0000-0003-0243-4606

alberto.abella@meloda.org

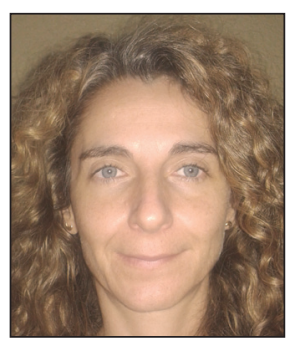

Marta Ortiz-de-Urbina-Criado es doctora en dirección y organización de empresas y profesora titular en la Universidad Rey Juan Carlos. Ha publicado artículos en revistas de impacto como International business review, International journal of technology management, Journal of knowledge management y Revista española de financiación y contabilidad, entre otras.

http://orcid.org/0000-0001-7527-6798

marta.ortizdeurbina@urjc.es

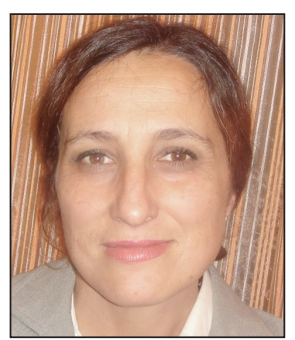

Carmen De-Pablos-Heredero es doctora en ciencias económicas y empresariales, profesora titular de universidad y directora del máster universitario en organización de empresas en la Universidad Rey Juan Carlos. Ha publicado artículos en revistas de impacto como CEDE, Universia, Interciencia, TIBE, Journal of entrepreneurship management, International journal of marketing research, Revista de ciencias sociales, Revista de economía mundial, Dyna, Pensée, Medical economics, REIS, etc. http://orcid.org/0000-0003-0457-3730

carmen.depablos@urjc.es

Universidad Rey Juan Carlos Facultad de Ciencias Jurídicas y Sociales Paseo de los Artilleros, s/n. 28032 Madrid, España

\section{Resumen}

Las políticas actuales para el aumento de la transparencia, la implantación del gobierno abierto o de las ciudades inteligentes tienen en la publicación de información uno de sus pilares fundamentales. Sin embargo, la disponibilidad de mecanismos de evaluación del uso y valor de esta información es limitada. Se presenta una nueva métrica, Meloda, que permite calificar la información y evaluar su grado de reutilización. Se describen las cuatro dimensiones de la métrica: estándares técnicos, acceso, legal, y modelo de datos, y se explica su proceso de evaluación.

\section{Palabras clave}

Datos abiertos, Reutilización de la información, Métrica, Gobierno abierto, Meloda.

\section{Title: Meloda, a metric to assess open data reuse}

\section{Abstract}

Current policies demanding an increase in transparency, open government and smart cities share open data publication as one of their basic pillars. However, there is a limited availability of mechanisms to assess the use and value of this information. The goal of this paper is to present a new metric, Meloda, that qualifies the information and helps to assess its reusability. Meloda's four dimensions are described: technical standards, access, legal considerations, and data model. Finally Meloda's assessment process is explained.

\section{Keywords}

Open data, Information reuse, Metric, Open government, Meloda. 
Abella, Alberto; Ortiz-de-Urbina-Criado, Marta; De-Pablos-Heredero, Carmen (2014). “Meloda, métrica para evaluar la reutilización de datos abiertos". El profesional de la información, v. 23, n. 6, noviembre-diciembre, pp. 582-588.

http://dx.doi.org/10.3145/epi.2014.nov.04

\section{Introducción}

La Open Government Partnership (Alianza para el Gobierno Abierto), nacida en 2011 y que incluye a 60 países (España desde abril de 2012), es una prueba del interés que el open government o gobierno abierto está suscitando en todo el mundo (Ramírez-Alujas; Dassen, 2014). Se trata de "una forma de comunicación abierta, permanente y bidireccional entre la administración y sus administrados, ciudadanos, empresas y sociedad civil" (Hofmann; Ramírez-Alujas; Bojórquez-Pereznieto 2012). Los tres pilares que lo definen son participación, colaboración y rendición de cuentas (publicación de información).

Las instituciones europeas están dando impulso a las políticas de reutilización de información como dinamizadoras de la economía digital y como base de la transparencia democrática.

Dentro del movimiento open data, que promueve la liberación de datos (Peset; Ferrer-Sapena; Subirats-Coll, 2011), se aboga porque las administraciones públicas pongan a disposición de la sociedad la información pública que tienen en su poder, a lo que se denomina open government data, para que cualquier persona u organización pueda, a partir de la misma, crear nuevas informaciones y servicios (Marcos-Martín; Soriano-Maldonado, 2011; Weiskopf; Weng, 2013). La apertura de la información del sector público permite establecer mecanismos de transparencia y favorece la colaboración ciudadana; aunque algunos autores apuntan a que la política de puesta a disposición de los datos no implica necesariamente que haya transparencia, ya que para lograrla es necesario que los datos estén tratados y presentados de tal forma que puedan reutilizarse para crear estudios, servicios de valor añadido o generar conocimiento para la sociedad (Porter; Millar, 1985; Shapiro; Varian, 1998; Ferrer-Sapena; Peset; Aleixandre-Benavent, 2011).

Reutilizando esta información se pueden ofrecer nuevos productos y servicios digitales, lo cual dinamiza la actividad económica y empresarial. Pueden crearse nuevas empresas que con pocos recursos propios lleven a cabo modelos de negocio basados en la reelaboración de dicha información, tratándola y añadiéndole valor (Marcos-Martín; SorianoMaldonado, 2011).

España está entrando en una dinámica activa en la liberación de datos públicos con la apertura de numerosos portales. EI localizador de datos abiertos a nivel mundial de la web de la Fundación CTIC ofrece un mapa de la situación en el que se incluyen 267 portales con conjuntos de datos públicos, de los cuales 32 son españoles. Sin embargo, hemos realizado una revisión de los portales locales, regionales y nacionales y hemos observado que esta cifra es superior a 50 .

http://datos.fundacionctic.org/sandbox/catalog/faceted

Hoy en día las ciudades inteligentes ${ }^{1}$ ofrecen una gran cantidad de información pública a través de sus portales. Esta información está recogida en catálogos que facilitan el acceso a las fuentes de información y que pueden ser utilizados para la creación de nuevos servicios por parte del sector privado, siendo, además, fuentes valiosas para los negocios de big data (LaValle et al., 2011; Kitchin, 2014).

La actualización de la Directiva 2003/98/CE relativa a la reutilización de la información del sector público en la Unión Europea, de junio de 2013 (Comisión Europea, 2013), confirma la importancia otorgada a este tema desde las más altas instancias políticas europeas. La puesta a disposición de información pública en España está regulada principalmente por la Ley 37/2007 de 16 de noviembre sobre reutilización de información (España, 2007), que tiene como objetivo "armonizar la explotación comercial de la información del sector público y la publicación de todos los documentos de libre disposición que obran en su poder, al considerar que son un instrumento esencial para el desarrollo del derecho al conocimiento, que constituye un principio básico de la democracia". Esta ley se complementa con el Esquema nacional de interoperabilidad ( $R D$ 4/2010, de 8 de enero) (España, 2010), así como por la reciente Ley de transparencia, acceso a la información y buen gobierno (Ley 19/2013, de 9 de diciembre) (España, 2013). Si bien otras normas, tanto nacionales como autonómicas y locales, condicionan aspectos o ámbitos de esta publicación.

Las políticas para el aumento de la transparencia, la implantación del gobierno abierto o de las ciudades inteligentes tienen en el open data uno de sus pilares fundamentales

La liberación de los datos supone un cambio organizativo, estructural y en la forma de trabajar de las administraciones, que tienen que establecer unas políticas de uso y privacidad sobre los que vayan a liberar, a cambio de ahorrar ciertos costes en la elaboración de los informes por parte de terceros (Ferrer-Sapena; Peset; Aleixandre-Benavent, 2011; LaValle et al., 2011). Sin embargo en muchos casos el sector público no tiene establecidas, o no es el objeto de su actividad, las rutinas organizativas que le permitan desarrollar la capacidad para extraer todo el valor del open data. Y es aquí donde las empresas pueden intervenir creando servicios de valor añadido que hagan la información más comprensible y asequible (Ferrer-Sapena; Peset; Aleixandre-Benavent, 2011; Lee; Kwak 2012).

Son todavía muy pocos los gobiernos o entidades públicas que puedan asegurar, o al menos estimar cuantitativamente los beneficios de sus políticas de apertura de datos públicos. En la mayoría de los casos sus estrategias de reutilización de datos se basan más en principios como el apoyo a las libertades ciudadanas, el cumplimiento de legislaciones propias, o declaraciones públicas de transparencia (Huijboom; Van-den-Broek, 2011). En España los informes elaborados 
por la administración pública a través de la entidad pública empresarial Red.es (Red.es, 2011; 2012) analizan el impacto del sector económico que reutiliza la información aunque no establecen una relación causal con sus políticas de publicación de datos.

Casi todas las iniciativas analizadas presentan una carencia de métricas tanto cuantitativas como cualitativas que puedan estimar adecuadamente su impacto. Fournier-Tombs (2011) explica que es técnicamente complicado el análisis del impacto general de los sitios de publicación de datos. Hace una revisión de los trabajos que han estudiado la influencia de los portales sobre la comunidad de usuarios y observa que los métodos basados en la medición del uso (encuestas sobre los reutilizadores y usuarios) son de utilidad limitada porque no se pueden obtener muestras estadísticamente significativas.

Por tanto se presenta como una necesidad estratégica disponer de métricas que evalúen la reutilización. Estas métricas pueden facilitar el uso eficiente de recursos de las administraciones públicas y servir a las empresas para conocer qué informaciones pueden tener un mayor potencial económico para la creación de negocios innovadores. El objetivo de este trabajo es explicar el desarrollo y utilidad de una nueva métrica que permite evaluar el grado de reutilización de esta información.

\section{Reutilización de datos}

La definición de reutilización según la RAE es "utilizar algo, bien con la función que desempeñaba anteriormente o con otros fines". La importancia de la reutilización de la información ya ha sido estudiada en otros muchos campos, desde el ámbito financiero (Chan; Greenbaum; Thakor, 1986), la medicina clínica (Kim, 2005) o la información científica (Faniel; Jacobsen, 2010).

La Ley 37/2007 (España, 2007) define la reutilización de la información del sector público como "el uso de documentos que obran en poder de las administraciones y organismos del sector público, por personas físicas o jurídicas, con fines comerciales o no comerciales, siempre que dicho uso no constituya una actividad administrativa pública". Se establecen 4 condiciones generales de reutilización:

- el contenido de la información no debe ser alterado;

- no se debe desnaturalizar el sentido de la información;

- se debe citar la fuente;

- se debe mencionar la fecha de la última actualización.

En este trabajo definiremos información reutilizable como aquella que cumple 4 condiciones mínimas que facilitan su uso:

1) no existencia de barreras técnicas a la reutilización;

2) posibilidad de acceso automatizado a la información (Eaves, 2010);

3) existencia de un esquema legal que permita su uso (Eaves, 2010);

4) acceso al conocimiento de la estructura de la información publicada.

Algunos autores han propuesto clasificaciones y formas de evaluar la calidad de la información pero no su reutilización.
Tabla 1. Principios del open government data http://opengovdata.org

\begin{tabular}{|l|l|}
\hline Complete & Acceso a toda la información \\
\hline Primary & No se accede a los datos de forma agregada \\
\hline Timely & Datos disponibles lo antes posible \\
\hline Accessible & $\begin{array}{l}\text { Datos accesibles para todos los propósitos y } \\
\text { tipos de usuarios }\end{array}$ \\
\hline Machine processable & $\begin{array}{l}\text { Procesamiento de datos de forma automá- } \\
\text { tica }\end{array}$ \\
\hline Non-discriminatory & $\begin{array}{l}\text { Datos disponibles para todos los usuarios } \\
\text { sin necesidad de registro }\end{array}$ \\
\hline Non-proprietary & Datos almacenados en un estándar abierto \\
\hline License-free & Datos no sujetos a restricciones en su uso \\
\hline
\end{tabular}

Por ejemplo, la clasificación en cinco estrellas de BernersLee $^{2}$ está orientada a evaluar si los datos están preparados para ser conectados (linked data) con otros conjuntos de datos. La métrica de Pipino, Lee y Wang (2002), que cuenta con 16 dimensiones de análisis, evalúa la calidad de la información, pero no sus posibilidades de reutilización. Ren y Glissmann (2012) analizan la arquitectura de datos de la organización para generar la publicación de datos abiertos con la mayor calidad de la información posible. Para ello presentan un proceso con 5 etapas para identificar la información relevante, comprobar su estado y diseñar los mecanismos de publicación. Sin embargo en su propuesta no se incluye la evaluación de la reutilización de esa información.

\section{La métrica Meloda permite calificar la información y evaluar su grado de reu- tilización}

\section{Meloda}

Nació en 2011 como reacción a la falta de homogeneidad en los conjuntos de datos que se publicaban en los portales open data, para analizar el grado de reutilización de la información. Para la elaboración de esta métrica se han considerado las tres leyes del open government data (Eaves, 2010):

- ser accesible por internet;

- ser legible por máquinas;

- que el marco legal permita usos distintos de los originales, así como los principios del open government data (tabla 1) (Lessig, 2007).

En las primeras versiones estables se consideraron tres dimensiones:

- Estándares técnicos. Trata de reflejar la importancia que tiene que la información sea almacenada en un estándar que no sea privativo (non-proprietary).

- Acceso a toda la información legible de forma automatizada, con el mayor detalle posible y para el mayor número de usos (complete, machine procesable, primary y accessible). 
Tabla 2. Meloda: dimensiones y niveles

\begin{tabular}{|c|c|c|c|}
\hline Estándares técnicos & Acceso & Legal & Modelo de datos \\
\hline $\begin{array}{l}\text { 1. Estándar privativo } \\
\text { Ej.: .xls, .shp, .doc }\end{array}$ & $\begin{array}{l}\text { 1. Sin acceso } \\
\text { Ej.: mail no automático o acceso } \\
\text { en persona }\end{array}$ & $\begin{array}{l}\text { 1. Copyright } \\
\text { Ej.: copyright }\end{array}$ & $\begin{array}{l}\text { 1. Sin modelo publicado } \\
\text { Ej.: tabla de datos sin descripción de los campos }\end{array}$ \\
\hline $\begin{array}{l}\text { 2. Estándar abierto } \\
\text { Ej.: .csv, .ods, wms }\end{array}$ & $\begin{array}{l}\text { 2. Acceso vía web con registro } \\
\text { Ej.: formulario manual }\end{array}$ & $\begin{array}{l}\text { 2. Uso privado } \\
\text { Ej.: copyright permitiendo } \\
\text { uso personal }\end{array}$ & $\begin{array}{l}\text { 2. Modelo con campos de datos } \\
\text { Ej.: tabla de datos con descripción de los campos }\end{array}$ \\
\hline \multirow[t]{3}{*}{$\begin{array}{l}\text { 3. Estándar abierto con } \\
\text { metadatos } \\
\text { Ej.: rdf, rss, json }\end{array}$} & $\begin{array}{l}\text { 3. Acceso directo vía web } \\
\text { Ej.: url único }\end{array}$ & $\begin{array}{l}\text { 3. Uso no comercial } \\
\text { Ej.: CC BY-NC } 4.0\end{array}$ & $\begin{array}{l}\text { 3. Modelo con especificaciones de campos } \\
\text { Ej.: vocabularios disponibles }\end{array}$ \\
\hline & $\begin{array}{l}\text { 4. Acceso vía web con paráme- } \\
\text { tros } \\
\text { Ej.: url con parámetros }\end{array}$ & $\begin{array}{l}\text { 4. Uso comercial } \\
\text { Ej.: CC BY-SA } 4.0\end{array}$ & $\begin{array}{l}\text { 4. Modelo externo normalizado } \\
\text { Ej.: vocabularios disponibles aceptados por } \\
\text { organización de normalización }\end{array}$ \\
\hline & $\begin{array}{l}\text { 5. Acceso completo (API) } \\
\text { Ej.: punto de acceso Sparql }\end{array}$ & $\begin{array}{l}\text { 5. Uso no limitado con } \\
\text { autoría } \\
\text { Ej.: CC BY } 4.0\end{array}$ & $\begin{array}{l}\text { 5. Modelo externo y generalizado } \\
\text { Ej.: vocabularios disponibles aceptados por } \\
\text { organización de normalización y reconocidos }\end{array}$ \\
\hline
\end{tabular}

- Legal. Importancia del marco legal en el uso de la información, y sin barreras legales para su uso, independientemente del respeto a la privacidad y a la seguridad (license-free).

Las primeras aplicaciones de la métrica se realizaron sobre 200 conjuntos de datos provenientes de portales de open data regionales, locales y nacionales de España. Se observó que era necesario incluir una cuarta dimensión que tuviera en cuenta el modelo de datos a publicar, que reflejara la importancia que tiene la estructura de datos para poder procesar la información (machine processable). La última versión, Meloda 3.10, incluye las cuatro dimensiones. http://bit.ly/meloda310

\subsection{Dimensiones de Meloda}

Las cuatro dimensiones que analiza son:

1) Estándares técnicos o estructura técnica en que se ofrece el dato. Califica las informaciones en tres niveles (tabla 2):

- 1: la información se ofrece en estándares privativos que incluyen restricciones de propiedad intelectual para su uso;

- 2: se alcanza cuando los datos están almacenados en formatos que no tienen restricciones para su utilización;

- 3: se alcanza cuando además del segundo nivel se incluye para cada dato un esquema de metadatos.

2) Acceso a la información o mecanismo por el cual se hace posible la descarga o conexión con la información. Presenta cinco niveles (tabla 2):

- 1: conjunto de datos que, o bien no tienen acceso web, o bien requieren una petición manual;

- 2: acceso mediante interacción manual del usuario o necesidad de registro;

- 3: url que sólo permite descargar el conjunto completo de datos;

- 4: al url se pueden añadir parámetros con los que no es necesario descargar o acceder a todo el conjunto de datos;

- 5: incluye aquellos conjuntos de datos que ofrecen la posibilidad de realizar consultas sobre los datos y cruzarlos con fuentes externas.

3) Marco legal: licencia que se asigna al conjunto de datos. Tiene cinco niveles (tabla 2):
- 1: el uso de la información se restringe completamente sin permiso explícito del autor;

- 2: se permite el uso de la información con fines particulares;

- 3: se permite el uso con fines no lucrativos;

- 4: se permite el uso comercial;

- 5: se permite un uso no limitado y la creación de derivados, siempre y cuando se reconozca la autoría.

La última versión, Meloda 3.10, incluye cuatro dimensiones: estándares técnicos, acceso a la información, marco legal y modelos de datos

4) Modelos de datos: modelo utilizado para publicar la información y la cantidad de veces que es utilizado en otras fuentes de datos. Tiene cinco niveles (tabla 2).

- 1: no se tiene un modelo publicado;

- 2: se identifican los campos de los datos;

- 3: se detallan y se publican las especificaciones de los campos que componen el modelo, aunque sea propio;

- 4: se usa un modelo externo normalizado, aunque esté poco extendido;

- 5: se usa un modelo generalizado.

La tabla 3 muestra la relación entre los niveles de las dimensiones de Meloda y la definición de open data (niveles en verde). Se puede observar que, aunque Meloda está centra-

Tabla 3. Relación entre dimensiones Meloda y open data

\begin{tabular}{|c|c|c|c|}
\hline Legal & Acceso & Estándares técnicos & Modelo datos \\
\hline 5 & 5 & 3 & 5 \\
\hline 4 & 4 & 2 & 4 \\
\hline 3 & 3 & 1 & 3 \\
\hline 2 & 2 & \multicolumn{2}{|c|}{} \\
\cline { 1 - 2 } & 1 & & 1 \\
\hline 1 & & & 2 \\
\hline
\end{tabular}


da en información open data, incluye también informaciones que no cumplen con la definición y la métrica puede ser de utilidad también en otros ámbitos.

\subsection{Proceso de evaluación de Meloda}

A continuación se describe el proceso de evaluación (figura 1). La tabla 4 describe los pesos que se asignan a cada uno de los niveles.

La puntuación final se calcula como la raíz cuarta del producto de las puntuaciones obtenidas en cada dimensión, ya que esta fórmula permite obtener una distribución más uniforme de los resultados. Además se puede analizar el efecto que tiene un cambio en la puntuación de una o más dimensiones sobre el grado de reutilización ${ }^{3}$.

La tabla 5 presenta los rangos de reutilización, entre 0 y 100 , que se proponen tras realizar una primera evaluación de los resultados con datos reales.

Meloda $=100 \times \sqrt[4]{\text { Estándar.técnico } \times \text { Acceso } \times \text { Legal } \times \text { Modelo } \cdot \text { datos }}$

A continuación se incluye un ejemplo de una evaluación realizada para un conjunto de datos con Meloda. La institución A publica un conjunto de datos como linked data pero con un modelo de datos publicado propio, con licencia de uso comercial, y con un mecanismo por el cual se puede utilizar un recurso Sparq/ para interrogar al conjunto de datos. Los niveles que tiene en cada dimensión son los siguientes:

$\begin{array}{lll}\text { Estándares técnicos } & \text { Nivel } 3 \text { (RDF con metadatos) } & 100 \% \\ \text { Acceso } & \text { Nivel 5 (Interrogable vía Sparql) } & 100 \% \\ \text { Legal } & \text { Nivel } 4 \text { (comercial) } & 90 \% \\ \text { Modelo de datos } & \text { Nivel 2 (modelo de datos ad hoc) } & 30 \%\end{array}$

Tabla 4. Ponderación de la métrica Meloda (\%)

\begin{tabular}{|c|c|c|c|c|c|c|}
\hline \# & Legal & Acceso & \# & Estándares técnicos & \# & Modelo datos \\
\hline 5 & 100 & 100 & \multirow{2}{*}{3} & \multirow{2}{*}{100} & 5 & 100 \\
\hline 4 & 90 & 90 & & & 4 & 90 \\
\hline 3 & 25 & 25 & \multirow[t]{2}{*}{2} & \multirow[t]{2}{*}{60} & 3 & 50 \\
\hline 2 & 10 & 10 & & & 2 & 10 \\
\hline 1 & 0 & 0 & 1 & 20 & 1 & 0 \\
\hline
\end{tabular}

Tabla 5. Rangos de reutilización

\begin{tabular}{|c|c|}
\hline Rangos & Calificación \\
\hline $75-100$ & Reutilización avanzada \\
\hline $50-75$ & $\begin{array}{l}\text { Reutilización avanzada con alguna característica } \\
\text { mejorable }\end{array}$ \\
\hline $25-50$ & Reutilización básica \\
\hline $0-25$ & Inadecuado para reutilización \\
\hline
\end{tabular}

$$
\text { Meloda }=100 \cdot \sqrt[4]{1 \times 1 \times 0,9 \times 0,3}=72,08
$$

Se observa que el conjunto de datos está en el rango de reutilización apta pero con alguna característica mejorable (tabla 5).

\section{Conclusiones}

En este trabajo se ha descrito una nueva métrica que caracteriza la reutilización a través de cuatro dimensiones y propone una forma cuantitativa para evaluarla. La última versión

(Meloda 3.10) se encuentra en proceso de mejora y actualización. Para el desarrollo de nuevas versiones se está considerando la inclusión de la dimensión temporal y geográfica y el ajuste de las ponderaciones de los distintos niveles.

Esta métrica tiene interés para todas las organizaciones que publican datos, públicas y privadas, y para todas las entidades, con o sin ánimo de lucro, que elaboran productos y servicios basados en ellas. Un tema interesante para futuros trabajos es el análisis del uso de la información que hacen las organizaciones (públicas, privadas, con ánimo de lucro o no), para identificar cuáles son las que crean más valor gracias a la reutilización. Los estudios previos sobre $\begin{array}{cc}\text { Paso 3. } & \text { Paso 4. } \\ \text { Obtención } & \text { Calificación } \\ \text { de valor } & \text { reutilización }\end{array}$

Figura 1. Proceso de evaluación de Meloda 
gobierno abierto suelen analizar las políticas gubernamentales y la creación de valor social (transparencia y colaboración ciudadana). Por otra parte, la literatura sobre reutilización de la información centra su atención en el uso de la información y en el análisis del valor económico que se puede crear mediante el uso eficiente de los recursos públicos -eficiencia- y/o de la creación de nuevos productos y servicios -innovación- (Jetzek; Avital; Bjørn-Andersen, 2013; 2014). Sin embargo, hay pocos trabajos que demuestren empíricamente el impacto económico de la reutilización de la información. Por ello, la investigación futura analizará, utilizando esta métrica, el valor económico que tiene la reutilización de la información.

\section{Notas}

1. Las ciudades inteligentes son un ecosistema públicoprivado que con uso intensivo de la tecnología proporciona servicios a los ciudadanos y a sus organizaciones.

2. Las cinco estrellas son: licencia abierta, datos estructurados, estándares abiertos, disponer de un identificador de recursos uniforme (URI) y fuentes conectadas.

http://5stardata.info

3. En las primeras versiones de la métrica se comprobó que la función suma de las dimensiones daba resultados incoherentes, y que la distribución uniforme de la puntuación de los niveles no permitía identificar bien los rangos de reutilización.

\section{Agradecimientos}

Este trabajo ha sido financiado por el proyecto ECO201236775 del Ministerio de Economía y Competitividad (España) y ha recibido el apoyo del proyecto Apps4EU financiado por The Information and Communication Technologies Policy Support Programme (ICI PSP).

\section{Bibliografía}

Chan, Yuk-Shee; Greenbaum, Stuart I.; Thakor, Anjan V. (1986). "Information reusability competition and bank asset quality". Journal of banking and finance, v. 10, n. 2, junio, pp. 243-253.

http://goo.gl/JX7OHi

http://dx.doi.org/10.1016/0378-4266(86)90008-7

Comisión Europea (2013). “Directiva 2013/37/UE del Parlamento Europeo y del Consejo, de 26 de junio de 2013, por la que se modifica la Directiva 2003/98/CE relativa a la reutilización de la información del sector publico". Diario oficial de la Unión Europea, 27 de junio.

https://www.boe.es/diario_boe/txt.php?id=DOUE-L-2013-81251

Eaves, David (2010). "The three laws of open government data". Conference for Parliamentarians: balancing openness and the public interest in protecting information.

http://bit.ly/eaves2010

España (2007). "Ley 37/2007, de 16 de noviembre, sobre reutilización de la información del sector público". Boletín oficial del estado, n. 276, 17 de noviembre, pp. 4716047165.

http://www.boe.es/buscar/doc.php?id=BOE-A-2007-19814
España (2010). "Real decreto 4/2010, de 8 de enero, por el que se regula el Esquema nacional de interoperabilidad en el ámbito de la administración electrónica". Boletín oficial del estado, n. 25, 29 de enero, pp. 8139-8156.

http://www.boe.es/boe/dias/2010/01/29/pdfs/ BOE-A-2010-1331.pdf

España (2013). "Ley 19/2013, de 9 de diciembre, de transparencia, acceso a la información y buen gobierno". Boletín oficial del estado, n. 295, 10 de diciembre, pp. 97922-97952. http://www.boe.es/boe/dias/2013/12/10/pdfs/ BOE-A-2013-12887.pdf

Faniel, Ixchel M.; Jocobsen, Trond E. (2010). "Reusing scientific data: How earthquake engineering researchers assess the reusability of colleagues' data". Computer supported cooperative work, v. 19, n. 3-4, August, pp. 355-375. http://dx.doi.org/10.1007/s10606-010-9117-8

Fournier-Tombs, Eleonore (2011). "Evaluating the impact of open data websites". Working papers series-SSRN. http://dx.doi.org/10.2139/ssrn.1926201

Ferrer-Sapena, Antonia; Peset, Fernanda; AleixandreBenavent, Rafael (2011). "Acceso a los datos públicos y su reutilización: open data y open government". El profesional de la información, v. 20, n. 3, mayo-junio, pp. 260-269. http://eprints.rclis.org/20997 http://dx.doi.org/10.3145/epi.2011.may.03

Hofmann, Andrés; Ramírez-Alujas, Álvaro; BojórquezPereznieto, José-Antonio (coords.) (2012). La promesa del gobierno abierto, Itiap.

http://www.lapromesadelgobiernoabierto.info

Huijboom, Noor; Van-den-Broek, Tijs (2011). “Open data: an international comparison of strategies". European journal of e-practice, n. 12, March-April, pp. 1-13.

http://www.epractice.eu/en/document/5290090

Jetzek, Thorhildur; Avital, Michel; Bjørn-Andersen, Niels (2013). "Generating value from open government data". En: $34^{\text {th }}$ Int l conf on information systems, Milan, pp. 1-20.

http://aisel.aisnet.org/cgi/viewcontent.cgi?article $=1181 \&$ co ntext=icis2013

Jetzek, Thorhildur; Avital, Michel; Bjørn-Andersen, Niels (2014). "Data-driven innovation through open government data". Journal of theoretical and applied electronic commerce research, v. 9, n. 2, May, pp. 100-120.

http://dx.doi.org/10.4067/S0718-18762014000200008

Kim, Hyeoneui (2005). Exploring data reusability: standardized representation of domain contents and feasibility testing. Doctoral dissertation, University of Minnesota, USA. ISBN: 0542310457

Kitchin, Rob (2014). "The real-time city? Big data and smart urbanism". GeoJournal, v. 79, Febr., pp. 1-14.

http://link.springer.com/article/10.1007\% 2 Fs10708-013-9516-8

LaValle, Steve; Lesser, Eric; Shockley, Rebecca; Hopkins, Michael S.; Kruschwitz, Nina (2011). "Big data, analytics and the path from insights to value". MIT Sloan management review, v. 52, n. 2, enero, pp. 21-32. 
http://sloanreview.mit.edu/article/big-data-analytics-andthe-path-from-insights-to-value

Lee, Gwanhoo; Kwak, Young-Hoon (2012). “An open government maturity model for social media-based public engagement". Government information quarterly, v. 29, Oct., pp. 492-503. http://dx.doi.org/10.1016/j.giq.2012.06.001

Lessig, Larry (2007). “Eight principles of open government data". Open government working group. http://www.opengovdata.org/home/8principles

Marcos-Martín, Carlos; Soriano-Maldonado, Salvador-Luis (2011). "Reutilización de la información del sector público y open data en el contexto español y europeo. Proyecto Aporta". El profesional de la información, v. 20, n. 3, mayo-junio, pp. 291-297.

http://www.elprofesionaldelainformacion.com/contenidos/2011/ mayo/07.pdf

http://dx.doi.org/10.3145/epi.2011.may.07

Peset, Fernanda; Ferrer-Sapena, Antonia; Subirats-Coll, Imma (2011). "Open data y linked open data: su impacto en el área de bibliotecas y documentación". El profesional de la información, v. 20, n. 2, marzo-abril, pp. 165-173.

http://www.elprofesionaldelainformacion.com/contenidos/2011/ marzo/06.pdf

http://dx.doi.org/10.3145/epi.2011.mar.06

Pipino, Leo L.; Lee, Yang W.; Wang, Richard Y. (2002). "Data quality assessment". Communications of the ACM, April, v. 45, n. 4, pp. 211-218.

http://web.mit.edu/tdqm/www/tdqmpub/ PipinoLeeWangCACMApr02.pdf

Porter, Michael E.; Millar, Victor E. (1985). "How information gives you competitive advantage". Harvard business re- view, v. 63, n. 4, pp. 149-160.

http://www.im.ethz.ch/education/HS11/MIS/docu/Porter85.pdf

Ramírez-Alujas, Álvaro; Dassen, Nicolás (2014). Vientos de cambio: El avance de las políticas de gobierno abierto en América Latina y el Caribe. Banco Interamericano de Desarrollo. Nota técnica \#idb-tn-629.

http://bit.ly/ramirez2014

Red.es (2011). Characterization study of the infomediary sector 2011.

http://datos.gob.es/datos/sites/default/files/files/aporta_ infomediary\%20sector_2011.pdf

Red.es (2012). Characterization study of the infomediary sector 2012.

http://www.ontsi.red.es/ontsi/sites/default/files/121001_ red_007_final_report_2012_edition_vf_en_1.pdf

Ren, Guang-Jie; Glissmann, Susanne (2012). “Identifying information assets for open data. The role of business architecture and information quality". En: Procs. of the IEEE $14^{\text {th }}$ Int l conf on commerce and enterprise computing, Sept., pp. 94-100.

http://doi.ieeecomputersociety.org/10.1109/CEC.2012.23

Shapiro, Carl; Varian, Hal R. (1998). Information rules. Harvard Business Press, Boston. ISBN: $087584863 \mathrm{X}$

Weiskopf, Nicole G.; Weng, Chunhua (2013). "Methods and dimensions of electronic health record data quality assessment: enabling reuse for clinical research". Journal of the American Medical Informatics Association, January, v. 20, n. 1, pp. 144-151.

http://www.ncbi.nlm.nih.gov/pmc/articles/PMC3555312

http://dx.doi.org/10.1136/amiajnl-2011-000681

\section{Nueva colección EPI Scholar}

\section{Libros académicos y científicos de Información y Documentación}

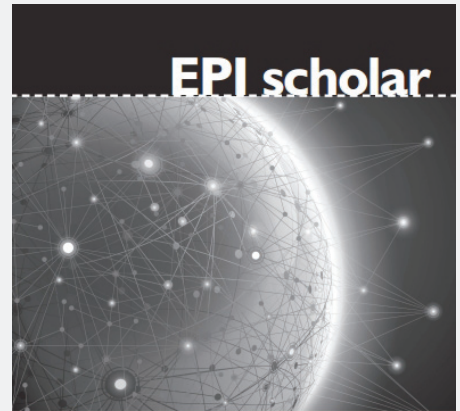

CIBERMETRÍA MIDIENDO EL ESPACIO RED ENRIQUE ORDUNA-MALEA ISIDRO F. AGUILLO

\section{Primer título: Cibermetría. Midiendo el espacio red de Enrique Orduña-Malea e Isidro F. Aguillo}

A pesar del crecimiento de la disciplina de la Cibermetría desde mediados de la década de los noventa, son escasos los libros académicos o manuales dedicados en exclusiva a la misma desde un contexto de las ciencias de la información y documentación. Este libro pretende cubrir este claro hueco en la literatura tanto nacional como internacional. 